\title{
Reaction-limited sintering in nearly saturated environments
}

\author{
Benny Davidovitch*, Deniz Ertaș, and Thomas C. Halsey \\ Corporate Strategic Research, ExxonMobil Research and Engineering, Route 22, Annandale, NJ 08801
}

\begin{abstract}
We study the shape and growth rate of necks between sintered spheres with dissolutionprecipitation dynamics in the reaction-limited regime. We determine the critical shape that separates those initial neck shapes that can sinter from those that necessarily dissolve, as well as the asymptotic evolving shape of sinters far from the critical shape. We compare our results with past results for the asymptotic neck shape in closely related but more complicated models of surface dynamics; in particular we confirm a scaling conjecture, originally due to Kuczinsky. Finally, we consider the relevance of this problem to the diagenesis of sedimentary rocks and other applications.
\end{abstract}

\section{INTRODUCTION}

Sintering is a surface-tension driven phenomenon in which solid particles packed below their melting temperature are consolidated via the growth of necks and subsequent shrinkage of pores between the particles. Quantitative understanding of sintering is crucial to study evolution of porous morphologies in industry and in nature - key properties of ceramic and metallic powders, like their strength and degree of compactness, are controlled by the sintering process (German, 1996; Herring, 1950; Kingerv and Berg, 1955; Kuczinskv, 1949). Packed snow flakes and ice spheres may be bonded together by necks well below their melting temperature - a phenomenon that is important for understanding snow avalanches and glacier flows (Colbeck, 1998; Hobbs and Mason, 1964; Kingerv, 1960; Kuroiwa, 1961; Maeono and Ebinuma, 1983). Sedimentary and other granular rocks also undergo sintering processes that affect their porosity and permeability (Hav and Evans, 1988; Jurewicz and Watson, 1985; Visser, 1999).

Sintering processes typically exhibit several stages, identified by the degree of porosity of the material and the nature of the dominant surface tension, as well as the kinetic route. In the early stage of sintering, when particles are barely touching each other, the driving force is the surface tension between the solid particles and the surrounding pore space - be it filled with vapor, solution, or vacuum. The chemical potential $\mu_{\text {sur }}(\mathbf{x})$ associated with surface tension at a point $\mathbf{x}$ on the surface is given by $\mu_{\text {sur }}(\mathbf{x})=\sigma \nu_{m} H(\mathbf{x})$, where $\nu_{m}$ is a molecular volume in the solid phase, $H=\left(\kappa_{1}+\kappa_{2}\right) / 2$ is the mean between the two principal curvatures $\kappa_{1,2}$, and $\sigma$ is the surface tension, here taken to be isotropic for simplicity. Typically, the contact zone between the particles is highly concave and has a negative $H$, whereas the particles themselves are convex with positive $H$. This difference in $H$, and hence in the chemical potential $\mu_{\text {sur }}(\mathbf{x})$, gives rise to a net flow of solid molecules from the pe-

\footnotetext{
${ }^{*}$ Current address: Division of Engineering and Applied Sciences, Harvard University, Cambridge, MA 02138
}

riphery to the touching zone and to the formation and growth of necks between the particles.

At later stages of the process, necks become thicker and variations in $H$ along the surface are diminished. Most of the pores are now concentrated inside grains and on grain boundaries, and the thermodynamic driving force for further compactification is not $\sigma$, but rather the grainboundary energy. The dominant kinetic mechanisms in these stages are associated mainly with bulk transport: grain-boundary diffusion, plastic flow and lattice diffusion (German, 1986; Swinkels and Ashbv, 1981).

Here we focus on early stage sintering where the mass transfer is controlled solely by dissolution-precipitation through a surrounding solution. Such kinetics is dominant when the solid phase is in coexistence with vapor or solution, and the temperature is not too close to the melting temperature, at which thermal energy is high enough to activate fast surface diffusion processes. In addition, we assume that thermodynamics (energy) and kinetics (diffusion) associated with grain boundaries can be neglected, as is the case, e.g., with amorphous materials. We will limit ourselves to cases in which the chemical reaction rate for dissolution-precipitation between the solid and solution is much slower than typical rates of molecular transport through the surrounding media. Under such reaction-limited dynamics spatial variations of $\mu_{\text {sol }}$, the chemical potential in the solution, can be neglected. Moreover, we will consider particles in solution under 'open' conditions, in which the total amount of solid material is not conserved, but the solute concentration, and hence $\mu_{\text {sol }}$, is fixed in time. These assumptions are unrealistic for industrial sintering of powders, in which proper inclusion of molecular transport and other considerations can be crucial.

The prototypical geometry for early stage sintering, introduced by Kuczinsky (Kuczinskv, 1949), and studied extensively since then, is of a cylindrical neck between two touching spherical particles. Assuming a characteristic shape for the evolving neck profile, it was shown (Herring, 1950; Kuczinskv, 1949), that for reaction-limited dynamics, dominated solely by dissolution-precipitation, the neck thickens in time as $t^{1 / 3}$ at short times. If diffusion through the vapor is much slower than the reaction rate, it was argued that the 
neck thickens as $t^{1 / 5}$ Hobbs and Mason, 1964). Other kinetic routes give rise as well to various power laws. During the last 50 years, many experiments aimed to test these theories, and power law growth rates of necks were indeed found (Hobbs and Mason, 1964; Kingerv, 1960; Kingerv and Berg, 1955). For sintering dominated by surface diffusion, several researchers argued, based on numerical simulations and analytic arguments, that Kuczinsky's growth rates are not correct (Nichols and Mullins 1965, German and Lathrop 1978, Eggers 1998). However, the multitude of microscopic mechanisms for sintering, and the fact that in real physical processes several of them may be significant, makes the verification of a simple theory a challenging task for the experimentalist.

In this paper we present a detailed study of sintering driven by our model dynamics in this geometry. We focus on two aspects of the surface dynamics: the critical surface, which is actually an unstable fixed point of the appropriate surface dynamics, and the asymptotic growth profile. Our analysis of the critical surface provides us with conditions on initial contact geometries that can become persistent sinters, and enables us to determine growth rates at very early stages of the sintering process. For the asymptotic profile, we verify the $t^{1 / 3}$ growth rate for the neck, derive analytically the correct neck profile, and explain how it selects the appropriate growth rate.

The paper is organized as follows: In section 2 we introduce our model system and derive an equation of motion for cylindrically symmetric surface evolution. In section 3 we study the structure of the unstable fixed point of this equation (the critical surface), and perform linear stability analysis of its dynamics. In section 4 we discuss the evolution of surfaces far from the critical surface, and show how the asymptotic dynamics can be described as a self-consistent solution of the equation of motion. In section 5 we conclude and suggest future directions. An appendix contains some calculational details.

\section{EQUATION OF SURFACE EVOLUTION}

Let us consider a solid in coexistence with its solution in a surrounding liquid (or vapor), and denote the interface between the two phases as $\Gamma \equiv \mathbf{x}(u, v)$. We assume a first order single-component chemical reaction between the solid and solution, characterized by $K_{f}$ - the dissolution rate of a flat solid interface to a 'fresh' (unsaturated) solution. We assume that the surface tension $\sigma$ between the two phases can be considered as isotropic (which will be true well above the roughening transition or for an amorphous solid). The normal velocity of the surface $u_{n}(\mathbf{x})$ into the fluid region is given by the difference between dissolution and precipitation rates

$$
u_{n}(\mathbf{x})=-K_{f}\left(1-e^{-\frac{\Delta \mu(\mathbf{x})}{k T}}\right),
$$

where the precipitation rate is controlled by the Boltzmann factor associated with the difference $\Delta \mu(\mathbf{x}) \equiv$ $\mu_{\text {sur }}(\mathbf{x})-\mu_{\text {sol }}(\mathbf{x})$ between the chemical potentials of solid and dissolved molecules on the two sides of the interface $\Gamma$. The chemical potentials $\mu_{\text {sur }}(\mathbf{x})$ and $\mu_{\text {sol }}(\mathbf{x})$ are given by

$$
\begin{aligned}
& \mu_{\text {sur }}(\mathbf{x})=2 \nu_{m} \sigma H(\mathbf{x})+\mu_{\text {flat }}, \\
& \mu_{\text {sol }}(\mathbf{x})=k T \log \frac{c(\mathbf{x})}{c_{\text {sat }}}+\mu_{\text {flat }},
\end{aligned}
$$

where $\mu_{\text {flat }}$ is the chemical potential of a flat surface in equilibrium with a saturated solution at concentration $c_{\text {sat }}, c(\mathbf{x})$ is the concentration near the surface point $\mathbf{x}$, $\nu_{m}$ is the molecular volume in the solid, and an ideal solution is assumed. Here and elsewhere we define all concentrations with respect to the concentration in the solid. Equation (2.2) follows from the geometrical identity $H(\mathbf{x})=(1 / 2)(\delta S / \delta V)$, relating the mean curvature and the local variation in surface area $\delta S$ with respect to a volume change $\delta V$ of the solid. For our system we assume that the surface tension energy is much smaller than $k T$, and that the solution is nearly saturated. Equation (2.1) then reduces to:

$$
u_{n}(\mathbf{x})=-K_{f} \frac{2 \nu_{m} \sigma}{k T}\left[H(\mathbf{x})-\frac{k T}{2 \nu_{m} \sigma} \log \frac{c(\mathbf{x}, t)}{c_{\mathrm{sat}}}\right] .
$$

In order to fully describe the evolution of a solid-fluid interface $\Gamma(t)$, equation (2.4) must be supplemented by an advection-diffusion equation governing the concentration in the solution, for which $c(\mathbf{x}, t)$ is the boundary value at the interface with the solid phase.

In this study we are interested in kinetic conditions for which any inhomogeneity in the concentration $c(\mathbf{x}, t)$ equilibrates much faster than the typical time for chemical reactions to cause significant changes of the surface shape by dissolution-precipitation events. In other words, we assume:

$$
\tau_{\text {diss }} \gg \tau_{\text {diff }} \quad \text { or } \quad \tau_{\text {diss }} \gg \tau_{\text {adv }},
$$

where the kinetic time scales are defined by

$$
\tau_{\text {diss }}=\frac{L_{\mathrm{par}}}{K_{f}}, \quad \tau_{\text {diff }}=\frac{L_{\text {pore }}^{2}}{D}, \quad \tau_{\text {adv }}=\frac{L_{\text {pore }}}{V},
$$

where $D$ is the diffusion constant, $V$ is typical velocity of the advecting flow, $L_{\mathrm{par}}$ is a typical size of a particle, and $L_{\text {pore }}$ is a typical separation between particles. Such kinetic conditions are typically realized in sedimentary rocks that dissolve extremely slowly, such that their surface morphology evolves over geological time scales.

Under this reaction-limited dynamics, we can assume a homogeneous distribution of the concentration and hence the chemical potential in the solution: $c(\mathbf{x}, t) \rightarrow c_{\infty}(t)$, fixed by conditions outside the local region of interest. The surface dynamics equation (2.4) then reduces to:

$$
\begin{aligned}
u_{n}(\mathbf{x}) & =-K_{f} \frac{2 \nu_{m} \sigma}{k T}\left[H(\mathbf{x})-H_{*}\right], \\
H_{*} & \equiv\left[k T /\left(2 \nu_{m} \sigma\right)\right] \log \left(c_{\infty}(t) / c_{\mathrm{sat}}\right) .
\end{aligned}
$$




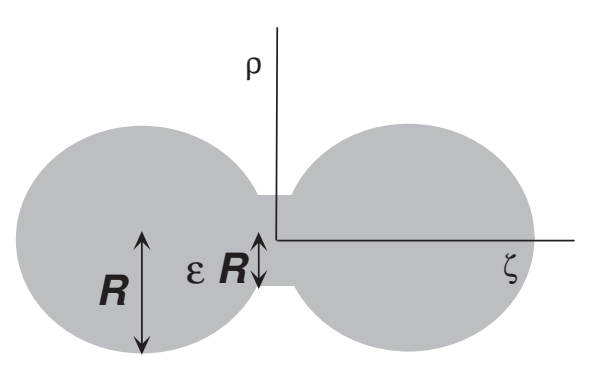

FIG. 1 Two sintering spheres-the sinter preserves the cylindrical symmetry around the $\zeta$-axis. The width of the neck divided by the diameter of the sphere defines the small parameter $\epsilon$.

For $H_{*}=0$ equation (2.7) becomes the well-known AllenCahn equation, describing the decay to global equilibrium of a binary system under the influence of surface tension (Brav, 1994).

In this paper we focus on the evolution under (2.7) of a sinter between spheres of radius $R$. Typical curvatures in the neck region are much higher (in absolute value) than the curvature of the spheres, and therefore we take $c_{\infty}(t)=c_{\mathrm{sat}} \exp \left(2 \nu_{m} \sigma / k T R\right)$, which guarantees that before contact is made the two solid spheres are in equilibrium with the solution. This condition replaces the more common condition of conservation of solid matter, which is usually encountered in applications in material science, and which can be implemented in the open, reactionlimited case by suitably varying $H_{*}$ (slowly) with time.

Depending on the surface geometry in the contact region, the dynamics (2.7) may lead to dissolution of the contact, or to sintering - a neck growing between the two spheres. Evolution of surfaces under equation (2.7) preserves cylindrical symmetry with respect to the axis connecting the centers of the two spheres.

Let us rewrite equation (2.7), taking advantage of this cylindrical symmetry. Defining the $\zeta$-axis as the axis of this symmetry, with $\rho(\zeta, t)$ the radial position of the surface (see Fig. (1), the in-plane (longitudinal) curvature $\kappa_{l}$ and the out-of-plane (azimuthal) curvature $\kappa_{a}$ are:

$$
\kappa_{l}=-\frac{\rho^{\prime \prime}}{\left[1+{\rho^{\prime}}^{2}\right]^{3 / 2}}, \quad \kappa_{a}=\frac{1}{\rho \sqrt{1+{\rho^{\prime}}^{2}}},
$$

yielding a mean curvature $H$ of

$$
H=\frac{1}{2}\left(\kappa_{a}+\kappa_{l}\right)=\frac{1}{2 \sqrt{1+{\rho^{\prime}}^{2}}}\left(\frac{1}{\rho}-\frac{\rho^{\prime \prime}}{1+{\rho^{\prime}}^{2}}\right) .
$$

Furthermore, the normal velocity $u_{n}$ is given by:

$$
u_{n}=\left(1+\rho^{\prime 2}\right)^{-1 / 2} \partial \rho / \partial t .
$$

Upon rescaling time and length by:

$$
t \rightarrow \frac{t}{k T /\left(R^{2} K_{f} \nu_{m} \sigma\right)}, \quad r=\rho / R, \quad z=\zeta / R,
$$

Equation (2.7) takes the form:

$$
\frac{\partial r}{\partial t}=-\left[\frac{1}{r}-\frac{r^{\prime \prime}}{1+r^{\prime 2}}-2 \sqrt{1+r^{\prime 2}}\right] .
$$

In the following sections we will study the surface dynamics described by equation (2.13).

\section{FIXED POINTS}

In this section we will discuss equation (2.13) near its fixed points - surfaces for which $\partial r / \partial t=0$ at each point. From equations (2.72.10) we see that such surfaces are cylindrically symmetric surfaces of constant mean curvature (CSCMC) whose mean curvature $H(z)=1$. First we discuss the structure of these surfaces, then we use linear stability analysis to study the dynamics near such surfaces.

\section{A. Structure}

According to equation (2.13), CSCMC are defined by solutions to the equation:

$$
2 \sqrt{1+r^{\prime 2}}=\frac{1}{r}-\frac{r^{\prime \prime}}{1+{r^{\prime}}^{2}} .
$$

This is a second order ordinary differential equation (ODE), and therefore two boundary conditions (BC) are required for a unique solution. Since we are interested here in sintering of two identical spheres, we consider the boundary conditions to be:

$$
r(z=0)=\epsilon, \quad r^{\prime}(z=0)=0,
$$

where $z=0$ is the middle point between the centers of the touching spheres, $\epsilon$ is the ratio between the neck size and the radius $R$ of the unsintered spheres (which determines $H_{*}$ ), and the latter condition reflects the symmetry between the two sides of the contact.

Equations (3.13.2) can be easily integrated numerically. The profiles $r(z)$ and their derivatives $r^{\prime}(z)$ for two values of $\epsilon$ are shown in figure 2, together with the profile $r_{s p h}(z)$ of a sphere of radius 1 , whose center lies on the $z$-axis at $z=1$. Obviously, $r_{s p h}^{\prime}(z)$ diverges as $z \rightarrow 0$.

The appearance of a singular inner region near $z=0$ in which $\left|r^{\prime}(z)-r_{s p h}^{\prime}(z)\right|>>O(\epsilon)$ indicates that a regular perturbative solution to equation (3.1) is not possible; this equation therefore calls for singular perturbation analysis.

\section{Singular perturbation analysis}

Another form of equation (3.1) is achieved by considering $z$ as a function of $r$ (assuming that the function 

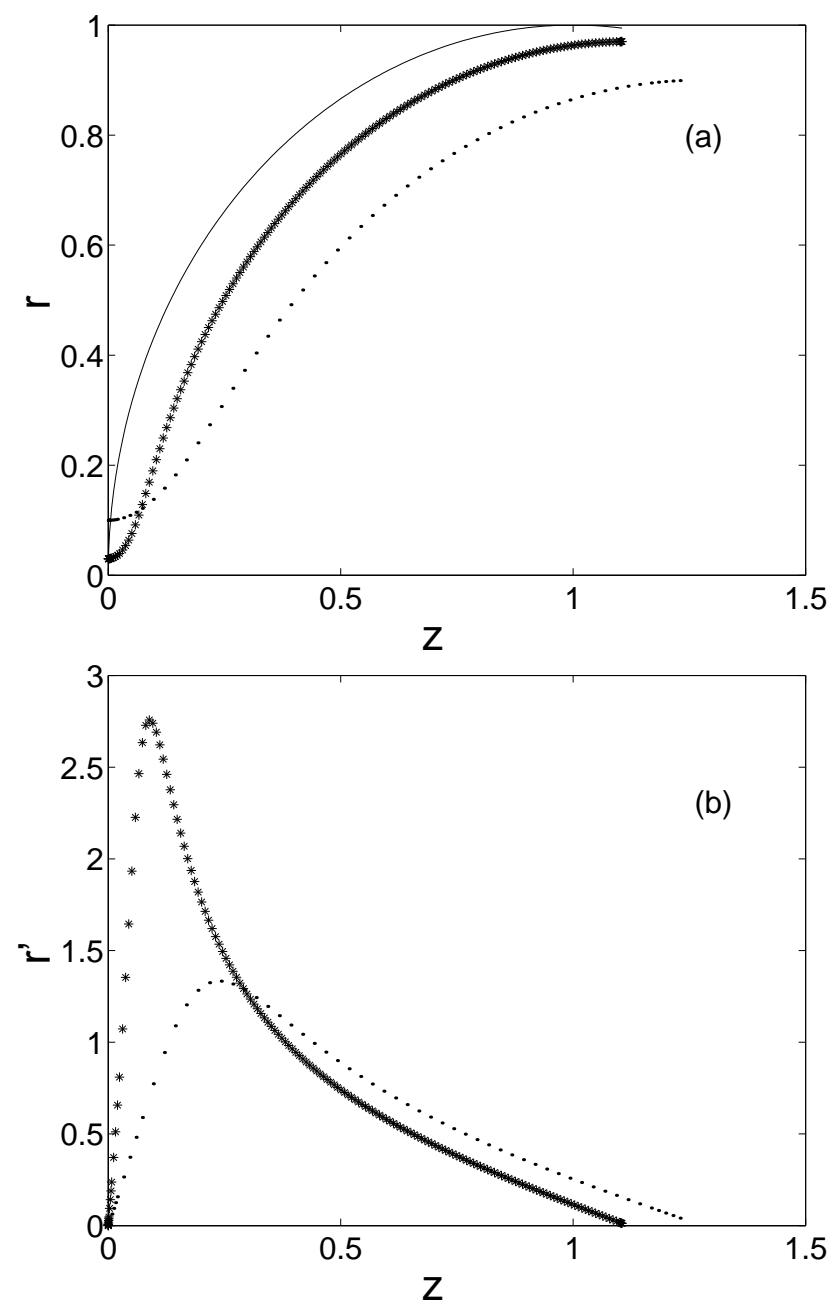

FIG. 2 Profile of a cylindrically symmetric constant mean curvature surface, obtained by numerical integration of equations (3.13.2), for two values of $\epsilon: \epsilon=0.1$ (dots), and $\epsilon=0.03$ (stars): (a) The profile $r(z)$. The solid curve is a spherical profile $r_{s p h}(z)$. (b) The derivative of the profile $r^{\prime}(z)$.

$r(z)$ is one-to-one):

$$
2 \sqrt{1+z^{\prime 2}}=\frac{z^{\prime}}{r}+\frac{z^{\prime \prime}}{1+z^{\prime 2}}
$$

and the transformation of the $\mathrm{BC}(\underline{3.2})$ is:

$$
z(r=\epsilon)=0, \quad z^{\prime}\left(r \rightarrow \epsilon^{+}\right) \rightarrow \infty .
$$

Equations (3.313.4) are more convenient for analytic study and will be used in the sequel instead of equations 3.13 .2 .

In order to construct a series expansion in $\epsilon$ for $z(r)$ in the interval $r \in[\epsilon, 1]$, one needs to consider separately the neck and the periphery, identify the small nondimensional parameters in each of them, and then match the two expansions.

Let us start with the peripheral region far from the neck, which we call the 'outer' region: $r \gg \epsilon$. In this region we can expand the profile $z_{\text {out }}(r)$ as a series

$$
z_{\text {out }}(r)=z_{\text {out }}^{(0)}(r)+\epsilon z_{\text {out }}^{(1)}(r)+O\left(\epsilon^{2}\right)
$$

where $z_{\text {out }}^{(0)}(r)$ is the profile of a sphere of radius 1 , centered on the $z$-axis at $(r=0, z=1)$ :

$$
z^{(0)}{ }_{\text {out }}(r)=1-\sqrt{1-r^{2}}
$$

We now consider the next order in $\epsilon$. Substituting in (3.3) the expansion (3.5) and the function $z_{\text {out }}^{(0)}$ (3.6), we obtain the following equation for $z_{\text {out }}^{(1)}$ :

$$
\frac{4 r^{2}-1}{r\left(1-r^{2}\right)}\left(z_{\text {out }}^{(1)}\right)^{\prime}=\left(z_{\text {out }}^{(1)}\right)^{\prime \prime}
$$

whose solution satisfies

$$
\left(z_{\text {out }}^{(1)}\right)^{\prime}=\frac{a}{r\left(1-r^{2}\right)^{3 / 2}},
$$

where $a$ is a constant of integration to be ultimately determined by a matching condition. Thus, to first order in $\epsilon$, the derivative of the outer solution is:

$$
z_{\text {out }}^{\prime}=\frac{r}{\sqrt{1-r^{2}}}+\epsilon \frac{a}{r\left(1-r^{2}\right)^{3 / 2}}+O\left(\epsilon^{2}\right) .
$$

A necessary condition for equation (3.5) to be a valid asymptotic expansion is that: $\left|\left(z_{\text {out }}^{(1)}\right)^{\prime}\right| \ll\left|\left(z_{\text {out }}^{(0)}\right)^{\prime}\right|$. This leads to the conditions:

$$
r \gg \sqrt{\epsilon}, \quad 1-r \gg \sqrt{\epsilon} .
$$

Condition (3.10) indicates the existence of an 'equatorial' region $1-\sqrt{\epsilon}<r<1$, where the outer perturbative expansion 3.5 is not valid, in which the profile is described by a different function $z_{e q u}(r)$ that matches $z_{\text {out }}(r)$. As we shall see below, this actually amounts to a displacement of the singularity at $r \rightarrow r_{s}=1$ in equations (3.6 3.8) to $r \rightarrow r_{s}<1$. Since our primary interest here is the neck structure, we postpone the analysis of $z_{\text {equ }}$ to the end of this section.

The divergence of $z^{\prime}$ required by condition (3.4) indicates that a regular expansion like (3.5) cannot satisfy equation (3.3) subject to the BC (3.4) (Hinch, 1991). This fact implies that a perturbative solution to equations (3.3 3.4 must be constructed from an expansion whose $0^{t h}$ order term is different from (3.6). We call this expansion $z_{\text {in }}(z)$, and write, similarly to equation (3.5):

$$
z_{i n}(r)=z_{i n}^{(0)}(r)+\epsilon z_{i n}^{(1)}(r)+O\left(\epsilon^{2}\right) .
$$

The function $z_{i n}(r)$ is required to satisfy equation (3.3) under the $\mathrm{BC}$ (3.4), and therefore we expect it to describe the profile near the contact between the two spheres.

The BC (3.4) indicates that a natural length scale at the neck region is $\epsilon$, and therefore we re-scale,

$$
\tilde{r}=\frac{r}{\epsilon}, \tilde{z}=\frac{z_{i n}}{\epsilon} .
$$


Equation (3.3) then becomes:

$$
2 \epsilon \sqrt{1+\left(\tilde{z}^{\prime}\right)^{2}}=\frac{\tilde{z}^{\prime}}{\tilde{r}}+\frac{\tilde{z}^{\prime \prime}}{1+\left(\tilde{z}^{\prime}\right)^{2}} .
$$

Assuming

$$
\epsilon \sqrt{1+\left(\tilde{z}^{\prime}\right)^{2}} \ll\left|\frac{\tilde{z}^{\prime}}{\tilde{r}}\right|,\left|\frac{\tilde{z}^{\prime \prime}}{1+\left(\tilde{z}^{\prime}\right)^{2}}\right|,
$$

and the expansion (3.11), we see that $\tilde{z}^{(0)}=z_{i n}^{(0)} / \epsilon$ satisfies the equation:

$$
\frac{\left(\tilde{z}^{(0)}\right)^{\prime}}{\tilde{r}}+\frac{\left(\tilde{z}^{(0)}\right)^{\prime \prime}}{1+\left(\tilde{z}^{(0)}\right)^{\prime 2}}=0,
$$

which is just the equation of a catenoid (CSCMC with zero mean curvature). The solution of equation (3.15) subject to the BC (3.4) satisfies:

$$
\left(\tilde{z}^{(0)}\right)^{\prime}=\frac{1}{\sqrt{\tilde{r}^{2}-1}} .
$$

Now let us calculate the first order term $z_{\text {in }}^{(1)}$ of the inner expansion. Substituting in equation (3.13) the expansion (3.11) and the catenoid function $\tilde{z}^{(0)}$, we get the following equation for $\tilde{z}^{(1)}=z_{\text {in }}^{(1)} / \epsilon$ :

$$
2 \frac{\tilde{r}}{\sqrt{\tilde{r}^{2}-1}}=\left(\frac{1}{\tilde{r}}+\frac{2}{\tilde{r}^{3}}\right)\left(\tilde{z}^{(1)}\right)^{\prime}+\frac{\tilde{r}^{2}-1}{\tilde{r}^{2}}\left(\tilde{z}^{(1)}\right)^{\prime \prime}
$$

whose solution satisfies:

$$
\left(\tilde{z}^{(1)}\right)^{\prime}=\frac{\tilde{r}^{2}}{\sqrt{\tilde{r}^{2}-1}} .
$$

The necessary condition $\left|\left(\tilde{z}^{(1)}\right)^{\prime}\right| \ll\left|\left(\tilde{z}^{(0)}\right)^{\prime}\right|$ implies $\tilde{r} \ll$ $1 / \sqrt{\epsilon}$, or

$$
r \ll \sqrt{\epsilon}
$$

which also establishes the validity of equation (3.14).

To first order in $\epsilon$, the inner expansion is thus

$$
\tilde{z}^{\prime}=\frac{1}{\sqrt{\tilde{r}^{2}-1}}+\epsilon \frac{\tilde{r}^{2}}{\sqrt{\tilde{r}^{2}-1}}+O\left(\epsilon^{2}\right) \text {. }
$$

From equations (3.10) and (3.19) we see that the inner and outer expansions are valid in non-overlapping regions in the interval $[\epsilon, 1]$. In order to match the two expansions $z_{\text {in }}$ and $z_{\text {out }}$, one has to look for a separate solution $z_{\text {mid }}$, valid in a 'middle' region around $r \sim \sqrt{\epsilon}$. Since the inner and outer expansions are valid at $r \ll \sqrt{\epsilon}$ and $r \gg \sqrt{\epsilon}$, respectively, a natural scaling for the variable and the function $z_{m i d}$ in the middle region is

$$
\tilde{\tilde{r}}=\frac{r}{\sqrt{\epsilon}}, \quad \tilde{\tilde{z}}=\frac{z_{m i d}}{\sqrt{\epsilon}} .
$$

The function $z_{\text {mid }}$ must match, to first order in $\epsilon$, both $z_{\text {in }}$ and $z_{\text {out }}$. This condition, and equations (3.9 3.20), imply the following form for the derivative $\tilde{\tilde{z}}^{\prime}$ :

$$
\tilde{\tilde{z}}^{\prime}(\tilde{\tilde{r}})=a \tilde{\tilde{r}}+\tilde{\tilde{r}}^{-1}+f(\tilde{\tilde{r}})+O\left(\epsilon^{2}\right),
$$

where asymptotically $f(\tilde{\tilde{r}})$ must satisfy:

$$
\begin{array}{rlll}
|f(\tilde{\tilde{r}})| \ll \tilde{r}^{-1} & \text { at } & & \tilde{r} \ll 1 \\
|f(\tilde{\tilde{r}})| \ll a \tilde{r} & \text { at } & & \tilde{r} \gg 1,
\end{array}
$$

in order to enable matching to $z_{\text {in }}$ and $z_{\text {out }}$. Substituting the form (3.22) in equation (3.3) and expanding to $O(\epsilon)$ we arrive at the solution for $f(\tilde{\tilde{r}})$ :

$$
f(\tilde{\tilde{r}})=\frac{1}{2} \epsilon\left(\tilde{\tilde{r}}^{3}+\tilde{\tilde{r}}^{-3}+3\right) .
$$

From equations 3.233.24 we see that the asymptotic expansion (3.22) for $z_{m i d}$ is valid in the region

$$
\sqrt{\epsilon} \ll \tilde{\tilde{r}} \ll \sqrt{\epsilon}^{-1}, \quad \text { or } \quad \epsilon \ll \mathrm{r} \ll 1 \text {. }
$$

From equations (3.93.103.193.203.223.243.25 we conclude that to first order in $\epsilon, z_{\text {mid }}(r)$ and $z_{i n}(r)$ match in the region $\epsilon \ll r \ll \sqrt{\epsilon}$, whereas $z_{\text {mid }}(r)$ and $z_{\text {out }}(r)$ match in the region $\sqrt{\epsilon} \ll r \ll 1$. The last matching condition also sets $a=1$.

To complete our first order asymptotic perturbation analysis we have to find $z_{e q u}(r)$ for $r \in(1-\sqrt{\epsilon}, 1]$. We note that unlike the $\mathrm{BC}$ (3.4), which gives rise to a new singularity on top of the spherical profile $z_{\text {out }}(r)$ in the neck region, the divergence of the outer expansion (3.5) for $1-r \sim \epsilon$, is caused simply because the original singularity, $z^{\prime}(r) \rightarrow \infty$ at $r_{s}=1$, is shifted to some $r_{s}(\epsilon)<1$. Analysis of equation (3.3) around the singular point shows that $z_{e q u}(r)$ satisfies:

$$
z_{\text {equ }}^{\prime}=c(\epsilon) / \sqrt{r_{s}(\epsilon)-r}, \quad 1-r \ll 1,
$$

where

$$
c(\epsilon)=1+O(\epsilon), \quad r_{s}(\epsilon)=1+O(\epsilon) .
$$

The functions $c(\epsilon)$ and $r_{s}(\epsilon)$ are determined from matching $z_{\text {equ }}$ to $z_{\text {out }}$ to $O(\epsilon)$ in the interval $\sqrt{\epsilon} \ll 1-r \ll 1$. Performing the matching procedure yields

$$
z_{\text {equ }}^{\prime}=\frac{1+\frac{1}{8} \epsilon}{\sqrt{2(1-\epsilon-r)}}+O\left(\epsilon^{2}\right)
$$

To conclude, to first order in $\epsilon$, the derivative $z^{\prime}(r)$ in the interval $(\epsilon, 1-\epsilon)$ is given by four different functional forms, equations (3.9 3.20 3.22 3.28), each valid in a different sub-interval of $\left(\epsilon, r_{s}(\epsilon)\right)$. These functions match along the overlaps between the sub-intervals. In terms of the variable $r$, they read 


$$
\begin{aligned}
z_{\text {in }}^{\prime}(r) & =\frac{\epsilon}{\sqrt{r^{2}-\epsilon^{2}}}+\frac{r^{2}}{\sqrt{r^{2}-\epsilon^{2}}}+\cdots ; \quad \text { inner region }: \epsilon<r \ll \sqrt{\epsilon} \\
z_{\text {mid }}^{\prime}(r) & =r+\frac{\epsilon}{r}+\frac{1}{2} r^{3}+\frac{1}{2} \frac{\epsilon^{3}}{r^{3}}+\frac{3}{2} \epsilon^{3 / 2}+\cdots ; \quad \text { middle region }: \epsilon \ll r \ll 1 \\
z_{\text {out }}^{\prime} & =\frac{r}{\sqrt{1-r^{2}}}+\epsilon \frac{1}{r\left(1-r^{2}\right)^{3 / 2}}+\cdots ; \quad \text { outer region }: \sqrt{\epsilon} \ll r<\ll 1-\sqrt{\epsilon} \\
z_{\text {equ }}^{\prime} & =\frac{1+\frac{1}{8} \epsilon}{\sqrt{2(1-r-\epsilon)}}+\cdots ; \quad \text { equatorial region }: 1-r \ll 1 .
\end{aligned}
$$

\section{Longitudinal stretching}

In order to get the actual profile $z(r)$, one has to integrate equations (3.2913.32). Since the four regions overlap each other along finite intervals, artificial limits of integration (within the overlapped intervals) must be introduced. Thus, we integrate (3.29) from $\epsilon$ to $\epsilon^{\alpha}$ $(1 / 2<\alpha<1)$, (3.30) from $\epsilon^{\alpha}$ to $\epsilon^{\beta}(0<\beta<1 / 2)$, (3.31) from $\epsilon^{\beta}$ to $1-\epsilon^{\gamma}(0<\gamma<1 / 2)$, and (3.32) from $1-\epsilon^{\gamma}$ to $1-\epsilon$. As will be verified below, the values of $\alpha, \beta, \gamma$ do not appear in the final expressions to leading order in $\epsilon$.

Performing the integrals of equations 3.2913 .32 between the above limits, we find the leading contributions to be:

$$
\begin{aligned}
& I_{1}=\int_{\epsilon}^{\epsilon^{\alpha}} z_{\text {in }}^{\prime} d r \approx(\alpha-1) \epsilon \log \epsilon+\cdots \\
& I_{2}=\int_{\epsilon^{\alpha}}^{\epsilon^{\beta}} z_{\text {mid }}^{\prime} d r \approx \frac{1}{2} \epsilon^{2 \beta}+(\beta-\alpha) \epsilon \log \epsilon+\cdots \\
& I_{3}=\int_{\epsilon^{\beta}}^{1-\epsilon^{\gamma}} z_{\text {out }}^{\prime} d r \approx 1-\sqrt{2} \epsilon^{\gamma / 2}-\frac{1}{2} \epsilon^{2 \beta}-\beta \epsilon \log \epsilon+\cdots \\
& I_{4}=\int_{1-\epsilon^{\gamma}}^{1-\epsilon} z_{\text {equ }}^{\prime} d r \approx \sqrt{2} \epsilon^{\gamma / 2}+\cdots
\end{aligned}
$$

In particular, the $z$-coordinates of the centers of the spheres (in practice, the 'equatorial' values of $z$ at which $d z / d r=0$ ) are shifted from \pm 1 (for $\epsilon=0$ ) to $\pm z_{s}=$ $\pm z\left(r_{s}(\epsilon)\right)$ :

$$
z_{s}=I_{1}+I_{2}+I_{3}+I_{4} \approx 1+\epsilon|\log \epsilon| .
$$

\section{B. Dynamics}

In a recent work (Davidovitch et al., 2002) we proved that all CMC surfaces, except the plane, are unstable under the dynamics (2.7). This holds in particular for the fixed point surfaces described by equations (3.29 3.32). Consider a surface $\mathbf{x}$ close to a fixed point surface $\mathbf{x}^{*}$ :

$$
\mathbf{x}\left(\mathbf{x}^{*}\right)=\mathbf{x}^{*}+\delta\left(\mathbf{x}^{*}\right) \mathbf{n}\left(\mathbf{x}^{*}\right),
$$

where $\mathbf{n}$ is a unit vector normal to the unperturbed surface, and $\delta$ is the magnitude of the perturbation. Linear stability analysis of the dynamics (2.7) near $\mathrm{x}^{*}$ results in the formal equation for the perturbation $\delta$ :

$$
\frac{\partial \delta}{\partial t}=\mathcal{L}\{\delta\}
$$

where $\mathcal{L}$ is a linear differential, generally non-Hermitian, operator, whose coefficients depend on the geometry of the surface $\mathbf{x}^{*}$. In this section we will compute, to leading order in $\epsilon$, the largest eigenvalue $\lambda_{\max }(\mathcal{L})$, for $\mathrm{x}^{*}$ a CSCMC whose profile $z(r)$ is described by equations (3.29 3.321). $\lambda_{\max }$ determines the rate of growth of a generic perturbation $\delta$ with a non-zero component along the maximal eigenvalue of equation (3.36), whether it leads to sintering of the spheres or to dissolution of the neck.

Derivation of the detailed form of $\mathcal{L}$ requires some tools of differential geometry, and will not be presented here. For our purposes it is enough to use the following result (Davidovitch et al., 2002): The eigenvalues of $\mathcal{L}$ are identical to the eigenvalues of a Hermitian operator $\mathcal{H}$ :

$$
\mathcal{H}=\frac{1}{2} \nabla_{s}^{2}+V\left(\mathbf{x}^{*}\right)
$$

where $\nabla_{s}$ is a surface gradient, and the 'potential' $V\left(\mathbf{x}^{*}\right)$ is:

$$
\begin{aligned}
V\left(\mathbf{x}^{*}\right) & =2 H_{*}^{2}-K-\frac{1}{2}\left[\nabla_{s}^{2} B+\left(\nabla_{s} B\right)^{2}\right] \\
B\left(\mathbf{x}^{*}\right) & \equiv-\frac{1}{8} \log \left(H_{*}^{2}-K\left(\mathbf{x}^{*}\right)\right),
\end{aligned}
$$

where $H_{*}, K=\kappa_{a} \kappa_{l}$ are respectively the mean and Gaussian curvatures of the unperturbed CMC surface. In particular, $\lambda_{\max }(\mathcal{L})=\lambda_{\max }(\mathcal{H})$. For a CSCMC with profile $z(r)$ we use the coordinate system $(\hat{r}, \hat{z}, \hat{\phi})$ to write:

$$
\begin{aligned}
\nabla_{s}^{2} & =\frac{1}{1+{z^{\prime}}^{2}} \nabla_{r}^{2}-\frac{z^{\prime} z^{\prime \prime}}{\left(1+{z^{\prime}}^{2}\right)^{2}} \nabla_{r}, \\
K(r) & =\frac{z^{\prime} z^{\prime \prime}}{r\left(1+{z^{\prime}}^{2}\right)^{2}} .
\end{aligned}
$$

We note that for the CSCMC described by equations (3.29 3.32), the Gaussian curvature $K(r)$ and its derivatives diverge toward the neck $(r \rightarrow \epsilon)$, as $\epsilon \rightarrow$ 
0 . This can be easily understood by recalling that $K=\kappa_{a} \kappa_{l}$. The largest value of the azimuthal curvature $\kappa_{a}=1 / r$ is clearly achieved at the neck, where $\kappa_{a, \text { neck }}=1 / \epsilon$. Since for this surface $\kappa_{l}=2-\kappa_{a} \approx-1 / \epsilon$, we see that $K_{\text {neck }} \approx-1 / \epsilon^{2}$ as $\epsilon \rightarrow 0$. As one moves away from the neck to the spherically shaped periphery, $\kappa_{a} \rightarrow 1$, the Gaussian curvature $K \rightarrow 1$, and therefore $|V|$ decreases. Thus, the maximal eigenvalue $\lambda_{\max }(\mathcal{H})$ is determined by the region near the neck, and we can compute it by considering the operator $\mathcal{H}$ in the inner region $\epsilon \leq r \ll \sqrt{\epsilon}$ only. According to equations (3.20 3.39), the change of variables:

$$
x=\sqrt{r^{2}-\epsilon^{2}},
$$

enables us to write $\nabla_{s}^{2}=\partial_{x}^{2}$ in the inner region. Some tedious algebra (see appendix) yields the potential form:

$$
V(x)=\frac{3}{4} \frac{\epsilon^{2}+\frac{1}{6} x^{2}}{\left(\epsilon^{2}+x^{2}\right)^{2}},
$$

in the inner region. With the $1 d$ potential (3.41), the eigenvalue equation for $\mathcal{H}$ is equivalent to a spheroidal wave equation (Abramowitz and Stegun, 1970). Numerical calculation of $\lambda_{\max }(\mathcal{H})$ in the limit $\epsilon \rightarrow 0$ yielded $\lambda_{\text {max }}(\mathcal{H})=0.282 \epsilon^{-2}+O\left(\epsilon^{-1}\right)$.

We conclude that the profile given by equations 3.29. 3.32) is an unstable fixed point of the dynamics (2.13). Typical perturbations will increase at a rate proportional to $\epsilon^{-2}$, and will lead either to sintering of the two spheres, or to dissolution of the neck.

This has some immediate consequences. Consider, for example, two spheres compressed against one another so that the distance between their centers is $d<2 R$. A sinter formed around the contact zone between such spheres will generally grow, since the contact geometry should typically be on the growth side of the critical neck geometry. On the other hand, if two spheres become connected by a neck while their centers are at a distance $d>2 R$, the dynamics of the neck may cause it to either grow or evaporate, the boundary between these two behaviors being approximated by $d \approx 2 R(1+\epsilon|\log \epsilon|)$.

\section{ASYMPTOTIC DYNAMICS}

In this section, we will study the asymptotic behavior of two sintering spheres when the surface profile is far from any fixed point, but the neck size is still small compared to the radius of the spheres. In this regime, the non-linear dynamics (2.13) cannot be approximated by its linearized form (3.36). In figure 3 we show the numerically computed evolution of an asymptotic neck profile, which appears to evolve according to a similarity law.

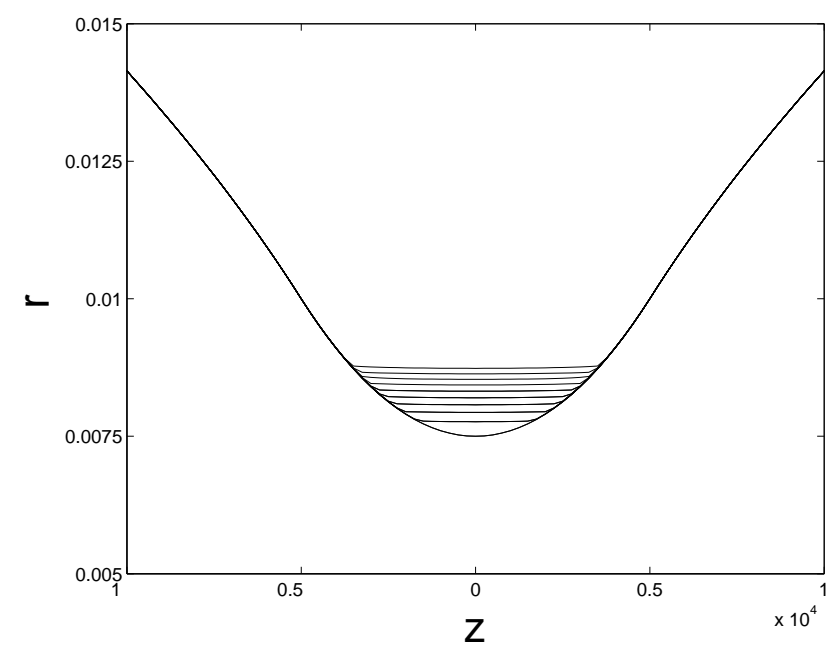

FIG. 3 Evolution of cylindrically symmetric sintering profile under equation (2.13). The initial profile is parabolic.

For volume preserving dynamics similar to (2.13), Kuczinsky (Kuczinskv, 1949) suggested that the asymptotic neck profile evolves like a spherical cup,

$$
r(z, t)=r_{0}(t)+w(t)-\sqrt{w^{2}(t)-z^{2}} ; \quad 0 \leq z \leq w(t),
$$

where $r_{0}(t)$ is the neck thickness and $w(t)$ its width. This neck profile has to be patched to the static peripheral spherical shape:

$$
r(z, t)=\sqrt{2 z-z^{2}}
$$

Patching the $z$-coordinates of the two regions at $z=$ $w(t)$ implies:

$$
\left(w(t)+r_{0}(t)\right)^{2} \approx 2 w(t) \Rightarrow w(t) \approx r_{0}(t)^{2} / 2 \ll r_{0}(t),
$$

where we used $r_{0}(t) \ll 1$. To obtain the time dependence of $r_{0}(t), w(t)$ we recall that the RHS of equation (2.13) is proportional to $\frac{1}{2}\left(\kappa_{a}+\kappa_{l}\right)-1$, where $\kappa_{a, \text { neck }} \approx 1 / r_{0}(t)$, and the spherical cup profile (4.1) implies $\kappa_{l} \approx-1 / w(t)$. According to equation (4.3) $w(t) \ll r_{0}(t) \ll 1$, and therefore the RHS of (2.13) is dominated by $w(t)^{-1}$, whereas the LHS is simply $d r_{0} / d t$. Using equation (4.3) we get

$$
\frac{d r_{0}}{d t} \approx \frac{1}{r_{0}^{2}} \Rightarrow r_{0} \sim t^{1 / 3},
$$

in agreement with experiments. A similar derivation for sintering dominated by surface diffusion yields $r_{0} \sim t^{1 / 7}$, and other growth rates can be derived for other kinds of kinetic routes (German, 1986; Herring, 1950; Kuczinskv, 1949; Swinkels and Ashbv, 1981).

It should be stressed here that Kuczinsky's profile is simply a guess and is not in any sense an asymptotic solution of equation (2.13) or of its volume preserving version. Moreover, notice that the $t^{1 / 3}$ growth rate cannot be derived for a general neck profile, but rather depends on 
the assumed shape. As an example, consider a parabolic neck profile:

$$
r(z, t)=r(t)+a(t) z^{2}
$$

Patching profiles and derivatives with the limit $z \rightarrow 0$ of (4.2) gives $r_{0} \sim t^{1 / 4}$.

Kuczinsky's assumption of semi-circular neck profile (4.1) was questioned by several authors, and other possible asymptotic neck profiles were suggested for sintering dominated by evaporation-precipitation and by other kinetic routes. In particular, several researchers (Amar et al., 1989; German and Munir, 1975), conjectured that in a variety of sintering processes, the neck profile evolves as a CSCMC surface, whose spatially constant mean curvature evolves in time. Notice the similarity between this assumption and Kuczinsky's one, according to which the longitudinal curvature $\kappa_{l}$ is spatially constant at the neck, whereas $\kappa_{a}$ may slightly vary. Other researchers, who focused their analysis on sintering dominated by surface diffusion, performed careful numerical simulations and advanced analytic arguments, from which they concluded that the asymptotic neck shape evolves very differently from the shape suggested by Kuczinsky, equation (4.1), (Eggers, 1998; German and Lathrop, 1978; Nichols and Mullins, 1965). Moreover, it was shown by these researchers that in that case the asymptotic growth rate of the neck is not $t^{1 / 7}$, as was suggested by Kuczinsky-like arguments for surface diffusion dynamics, but rather becomes closer to $t^{1 / 6}$ or even $t^{1 / 5}$. Other studies, which focused on kinetics dominated by surface and grain boundary diffusion showed that the neck profile and its growth rate in this case are also much more complicated than the simple models suggested by Kuczinsky (Bross and Exxner, 1979; Swinkels and Ashbv, 1980).

Here we show that the asymptotic neck profile for the sintering process determined by equation (2.13) evolves to a similarity shape, which is indeed different from Kuczinsky's or other suggested shapes. We show however, that Kuczinsky's $t^{1 / 3}$ growth rate is correct in this case.

Let us write the sinter profile $r(z, t)$ as

$$
r(z, t)=r_{0}(t)+b(z, t),
$$

with $b(0)=0$, so that $r_{0}(t)$ is simply the radius at the center of the sinter. We suppose that throughout the sintering, non-spherical, region, we can assume

$$
b(z, t) \ll r_{0}(t),
$$

an assumption we must verify for self-consistency at the conclusion of the calculation. Then we have

$$
\frac{b^{\prime \prime}}{1+b^{\prime 2}} \approx 2 \dot{r_{0}}+\frac{1}{r_{0}} \equiv \alpha(t)
$$

where we have replaced $r$ by $r_{0}$ and $2 \sqrt{1+{r^{\prime}}^{2}} \approx 0$, their values at $z=0$, while retaining the longitudinal curva- ture term (which also dominates the curvature in Kuczinsky's solution). The solution of equation (4.8) is:

$$
b(z, t) \approx-\frac{1}{\alpha(t)} \log \cos [\alpha(t) z]
$$

where we assumed the boundary condition: $b^{\prime}(0)=0$ (from $z \leftrightarrow-z$ symmetry).

Following a similar reasoning to the one presented in the beginning of this section, we notice that patching derivatives of the concave neck profile given by equations (4.64.9), and the convex spherical periphery, equation (4.2) is possible only for $(r, z) \rightarrow\left(r^{*}, z^{*}\right)$ where both derivatives become much larger than one:

$$
\left.\frac{\partial r}{\partial z}\right|_{z^{*}} \approx \tan \left[\alpha(t) z^{*}\right] \approx \frac{1}{\pi / 2-\alpha(t) z^{*}} \approx \frac{1}{\sqrt{2 z^{*}}}
$$

from which we get:

$$
z^{*}=\frac{\pi}{2 \alpha(t)}+O\left(\alpha^{-3 / 2}\right)
$$

Matching to the peripheral spherical shape equation (4.2) implies:

$$
r^{*} \approx \sqrt{2 z^{*}}
$$

From equations 4.84.114.12 we get:

$$
2 \dot{r_{0}} \approx-\frac{1}{r_{0}}+\frac{\pi}{r_{0}^{2}} \approx \frac{\pi}{r_{0}^{2}}
$$

whose asymptotic solution [at $r_{0}(t=0) \ll r_{0}(t) \ll 1$ ] gives the Kuczinsky growth rate (4.4),

$$
r_{0}(t) \approx\left(\frac{3 \pi t}{2}\right)^{1 / 3}
$$

To check the consistency of this solution we must verify the validity of the approximation $\left|b(z, t) / r_{0}(t)\right| \ll 1$ for $z<z^{*}$. This seems clear, since although $b^{\prime}$ is large at the patching point for small $z^{*}, b$ is still small $\left(b \ll r_{0}\right)$ because of equations 4.94.11. However, while inside the neck, near $z=0$, the term $2 \sqrt{1+z^{\prime 2}}$ (excluded from equation (4.8) is negligible, we should insure that it does not become large as $z \rightarrow z^{*}$. Therefore this term should be included in the consistency check, and we seek a solution to the equation:

$$
\frac{b^{\prime \prime}}{1+b^{\prime 2}}+2 \sqrt{1+b^{\prime 2}}=2 \dot{r}_{0}+\frac{1}{r_{0}}=\alpha(t),
$$

under the same BC as above: $b(0)=b^{\prime}(0)=0$. With the change of variables: $\tan u=b^{\prime}$, equation (4.15) recasts into the form:

$$
u^{\prime}+2 \sqrt{1+\tan ^{2} u}=\alpha(t)
$$


from which we get

$$
\begin{aligned}
b\left(z^{*}\right) & =\int_{0}^{z^{*}} d z b^{\prime}=\int_{0}^{\tan ^{-1}\left[b^{\prime}\left(z^{*}\right)\right]} d u \frac{\tan u}{\alpha(t)-2 \sqrt{1+\tan ^{2} u}} \\
& \approx \frac{1}{2 \alpha(t)} \log \left(1+\left[b^{\prime}\left(z^{*}\right)\right]^{2}\right) .
\end{aligned}
$$

This result is identical for small $z^{*}$ to equation (4.9), from which we verify the consistency of equation (4.10).

\section{CONCLUSIONS}

In this paper we studied a sintering model that we believe to be relevant for understanding the evolution of morphologies in sedimentary rocks and other geophysical systems, under reaction-limited kinetics and open environmental conditions. The assumption of reaction as opposed to transport limited kinetics is quite defensible in this context. For example, for siliciclastic rocks (quartz in water) we take as representative values: $L_{\text {par }} \approx L_{\text {pore }} \approx 10^{-4} \mathrm{~m}, D \approx 10^{-9} \mathrm{~m}^{2} / \mathrm{s}$, and $K_{f} \approx 210^{-15} \mathrm{~m} / \mathrm{s}$. From equation (2.6) we obtain the typical time scales $\tau_{\text {diss }} \approx 10^{11} \mathrm{~s} \gg \tau_{\text {diff }} \approx 10 \mathrm{~s}$. However, much of the diagenesis of sedimentary rocks is driven by pressure solution, rather than by surface energy effects (de Boer, 1977; Rutter, 1976). Surface energy effects might dominate in systems in which the solid phase has not experienced significant pressure changes over the lifetime of the grains-a situation that might prevail in magmatic plumes, in which solidified material coexists with liquid magma still saturated with the components of this material (Visser, 1999).

In glaciology, the most likely complication to our assumption of dissolution-precipitation kinetics driven by surface energies is likely to be surface melting, especially near the melting temperature of the ice. Transport via surface diffusion or in melted surface layers is also likely to be significant for sintered metallic powders, especially if the vapor pressure of the metals is low.

Even though the sintering physics of real technological and geological materials is likely rarely to be in a regime where the rather simplified model of this work includes all of the relevant physics, we nevertheless believe that our methods, based on careful asymptotic analysis of the stationary states of the kinetics, combined with similarity analysis of late-stage sintering, not only illuminate the physical limit we have chosen, but also should deal successfully with other types of tranport mechanisms. This is likely to be the subject of subsequent works.

\section{References}

Abramowitz, M., and I. Stegun, 1970, Handbook of Mathematical Functions (Dover, New York).

Amar, F., J. Bernholc, R. Berry, J. Jellinek, and P. Salamon, 1989, J. Appl. Phys. 65, 3219.

de Boer, R., 1977, Geochim. Cosmochim. Acta 41, 249.

Bray, A., 1994, Adv. Phys. 43, 357.

Bross, P., and H. Exxner, 1979, Acta Metall. 27, 1013.

Colbeck, S., 1998, J. Appl. Phys. 84, 4585.

Davidovitch, B., D. Ertaş, and T. Halsey, 2002, condmat/0210503.

Eggers, J., 1998, Phys. Rev. Lett. 80, 2634.

German, R., 1986, Liquid Phase Sintering (Plenum Publishing, New York).

German, R., 1996, Sintering Theory and Practice (John Wiley and Sons, New York), 1st edition.

German, R., and J. Lathrop, 1978, J. Mater. Sci. 13, 921.

German, R., and Z. Munir, 1975, Metal. Trans. B 6B, 289.

Hay, R., and B. Evans, 1988, J. Geophys. Res. 93, 8959.

Herring, C., 1950, J. Appl. Phys. 21, 301.

Hinch, E., 1991, Perturbation Methods (Cambridge University Press, Cambridge).

Hobbs, P., and B. Mason, 1964, Phil. Mag. 8, 181.

Jurewicz, S., and E. Watson, 1985, Geochim. Cosmochim. Acta 49, 1109.

Kingery, W., 1960, J. Appl. Phys. 31, 833.

Kingery, W., and M. Berg, 1955, J. Appl. Phys. 26, 1205.

Kuczinsky, G., 1949, J. Metal. 1, 169.

Kuroiwa, D., 1961, Tellus 12, 252.

Maeono, N., and T. Ebinuma, 1983, J. Phys. Chem. 87, 4103.

Nichols, F., and W. Mullins, 1965, J. Appl. Phys. 36, 1826.

Rutter, E. H., 1976, Phil. Trans. R. Soc. Lond. A 283, 203.

Swinkels, F., and M. Ashby, 1980, Powder Metall. 1, 1.

Swinkels, F., and M. Ashby, 1981, Powder Metall. 29, 259.

Visser, H., 1999, Mass transfer processes in crystalline aggregates containing a fluid phase, Ph.D. thesis, Utrecht University.

\section{APPENDIX}

In this appendix we prove that the potential $V$, as defined in equation (3.38) has the form (3.41) in the inner region $z_{i n}(r)$. For the CSCMC surface (3.29 3.32) $H=1$, $\nabla_{s} H=0$, and from equation (3.38) we get:

$$
V=2-K-\frac{1}{16} \frac{\nabla_{s}^{2} K}{1-K}-\frac{9}{128} \frac{\left(\nabla_{s} K\right)^{2}}{(1-K)^{2}}
$$

From equation (3.39) we get: 


$$
\begin{aligned}
& \left|\nabla_{s} K\right|=\frac{1}{r\left(1+{z^{\prime}}^{2}\right)^{5 / 2}}\left[z^{\prime \prime 2}+z^{\prime} z^{\prime \prime \prime}-\frac{z^{\prime} z^{\prime \prime}}{r}-\frac{4{z^{\prime}}^{2}{z^{\prime \prime}}^{2}}{1+{z^{\prime}}^{2}}\right] \\
& \nabla_{s}^{2} K=\frac{1}{r\left(1+z^{2}\right)^{3}} \quad\left[3 z^{\prime \prime} z^{\prime \prime \prime}+z^{\prime} z^{\prime \prime \prime \prime}+2 \frac{z^{\prime} z^{\prime \prime}}{r^{2}}-2 \frac{z^{\prime} z^{\prime \prime \prime}}{r}-2 \frac{z^{\prime \prime 2}}{r}\right. \\
& \left.-13 \frac{z^{\prime} z^{\prime \prime 3}}{1+{z^{\prime}}^{2}}-13 \frac{z^{\prime 2} z^{\prime \prime} z^{\prime \prime \prime}}{1+{z^{\prime}}^{2}}+28 \frac{{z^{\prime}}^{3}{z^{\prime \prime}}^{3}}{\left(1+{z^{\prime}}^{2}\right)^{2}}+9 \frac{z^{\prime 2} z^{\prime \prime 2}}{r\left(1+z^{\prime 2}\right)}\right] .
\end{aligned}
$$

Consider now the profile $z_{i n}(r)$ in the inner region $\epsilon \leq$ $r \ll \sqrt{\epsilon}$, as given in equation (3.29). Since in this region $r^{2} /\left(r^{2}-\epsilon^{2}\right) \ll 1$, we find that the leading contributions to the profile derivatives are:

$$
\begin{aligned}
z^{\prime} & =\frac{\epsilon}{\left(r^{2}-\epsilon^{2}\right)^{1 / 2}}, & z^{\prime \prime} & =-\frac{\epsilon r}{\left(r^{2}-\epsilon^{2}\right)^{3 / 2}}, \\
z^{\prime \prime \prime} & =\frac{2 \epsilon r^{2}+\epsilon^{3}}{\left(r^{2}-\epsilon^{2}\right)^{5 / 2}}, & z^{\prime \prime \prime \prime} & =-\frac{6 \epsilon r^{3}-9 r \epsilon^{3}}{\left(r^{2}-\epsilon^{2}\right)^{7 / 2}} \cdot(5.3)
\end{aligned}
$$

Substituting the last expressions for the profile derivatives in equation (5.2), and using the change of variables (3.40), we obtain equation (3.41). 